\title{
A modified strong-contrast expansion for estimating the effective thermal conductivity of multiphase heterogeneous materials
}

Masoud Safdari, Majid Baniassadi, Hamid Garmestani, and Marwan S. Al-Haik

Citation: Journal of Applied Physics 112, 114318 (2012); doi: 10.1063/1.4768467

View online: http://dx.doi.org/10.1063/1.4768467

View Table of Contents: http://scitation.aip.org/content/aip/journal/jap/112/11?ver=pdfcov

Published by the AIP Publishing

\section{Articles you may be interested in}

Materials with constant anisotropic conductivity as a thermal cloak or concentrator

J. Appl. Phys. 117, 054904 (2015); 10.1063/1.4907219

Cross-plane thermal conductivity of a $\mathrm{PbSnSe} / \mathrm{PbSe}$ superlattice material

Appl. Phys. Lett. 99, 041903 (2011); 10.1063/1.3615797

Thermal conductivity of chalcogenide material with superlatticelike structure

Appl. Phys. Lett. 98, 101904 (2011); 10.1063/1.3562610

Effective thermal conductivity of polycrystalline materials with randomly oriented superlattice grains

J. Appl. Phys. 108, 034310 (2010); 10.1063/1.3457334

Strong-contrast expansions and approximations for the effective conductivity of isotropic multiphase composites J. Appl. Phys. 94, 6591 (2003); 10.1063/1.1619573

MIT LINCOLN

LABORATORY CAREERS

Discover the satisfaction of innovation and service

to the nation
- Space Control

- Air \& Missile Defense

- Communications Systems \& Cyber Security

- Intelligence, Surveillance and

Reconnaissance Systems

- Advanced
Electronics
" Tactical Systems
- Homeland
Protection
- Air Traffic Control

LINCOLN LABORATORY

MASSACHUSETTS Institute OF TeChNOLOGY

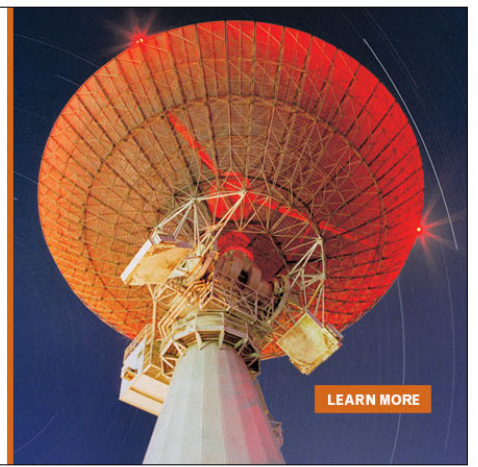




\title{
A modified strong-contrast expansion for estimating the effective thermal conductivity of multiphase heterogeneous materials
}

\author{
Masoud Safdari, ${ }^{1}$ Majid Baniassadi, ${ }^{2}$ Hamid Garmestani, ${ }^{3}$ and Marwan S. Al-Haik ${ }^{1, a)}$ \\ ${ }^{1}$ Engineering Science and Mechanics Department, Virginia Tech, MC 0219, Blacksburg, Virginia 24061, USA \\ ${ }^{2}$ School of Mechanical Engineering, College of Engineering, University of Tehran, P.O. Box 11155-4563, \\ Tehran, Iran \\ ${ }^{3}$ School of Materials Science and Engineering, Georgia Institute of Technology, 771 Ferst Drive N.W., \\ Atlanta, Georgia 30332, USA
}

(Received 23 July 2012; accepted 6 November 2012; published online 7 December 2012)

\begin{abstract}
To evaluate the effective thermal conductivity of a general anisotropic multiphase microstructure, a modified version of statistical strong-contrast expansions is formulated here. The proposed method takes into account the shape, orientation, and distribution of each phase through two-point and threepoint correlation functions. By applying a recently developed method, three-point correlation functions are approximated from the two-point correlation functions. Numerically, it is shown that for high contrast constituents, the solution of the third-order strong-contrast expansions is very sensitive to the selection of the reference medium. A technique is proposed to minimize the sensitivity of the solution. To establish the validity of the methods developed, the effective thermal conductivity of a number of isotropic and anisotropic two-phase and three-phase microstructures is evaluated and compared to their corresponding finite element (FE) simulations. Good agreement between the FE simulations and the proposed method predictions in the cases studied confirms its validity. When there are orders of magnitude disparity between the properties of the constituents, the developed method can be applied to better estimate the effective thermal conductivity of the multiphase heterogeneous materials in comparison with previous strong contrast model and other homogeneous methods. (C) 2012 American Institute of Physics. [http://dx.doi.org/10.1063/1.4768467]
\end{abstract}

\section{INTRODUCTION}

With the introduction of nanomaterials, many investigations have been devoted to incorporate them into polymer composites as fillers. ${ }^{1}$ Carbon nanotubes (CNTs) and graphite nanoplatelets (GNPs) are two nanostructured allotropic forms of carbon with intrinsically elevated thermal conductivity. ${ }^{1,2}$ When utilized as particulate nanofillers, they can dramatically enhance the effective thermal conductivity of their derivative nanocomposites. A number of reference closed form relations are developed based on classical homogenization techniques to evaluate the effective thermal conductivity of the composites materials. ${ }^{3}$ However, most of these relations are not applicable for nanocomposite in which the filler phases usually exhibit large thermal conductivity compared to the matrix, shape polydispersity (multiphase), and random heterogenous structure (nonperiodic).

Classic homogenization techniques can be classified into statistical methods, ${ }^{4}$ mean field methods, ${ }^{5}$ asymptotic methods, ${ }^{6}$ variational energy-based methods, ${ }^{7}$ and empirical/ semiempirical methods. ${ }^{8}$ Generally, the mean field homogenization and the asymptotic approaches are restricted to the periodic particular inclusion based structures and, therefore, they are not applicable for the homogenization of the random heterogeneous materials. Variational energy based methods typically provide an upper-bound or a lower-bound on the effective properties under specific conditions. ${ }^{3,4}$ However,

\footnotetext{
${ }^{\text {a) }}$ Author to whom correspondence should be addressed. Electronic mail: alhaik@vt.edu.
}

statistical homogenization techniques are neither limited to the periodic microstructures nor just providing bounds on the effective properties. In practice, statistical continuum theory provides tool for mathematical depiction of the dispersion and distribution of the heterogeneous materials. ${ }^{9}$ In these methods, structural information is usually transmitted in the solution through microstructural descriptor functions which are appearing within the solution expression. ${ }^{4}$ Thus, by utilizing these methods, the properties of the heterogeneous materials could be directly linked to the morphological details through different types of correlation functions such as the N-point correlation functions., ${ }^{4,9}$

According to the statistical continuum theory, the distribution, orientation, and the shape of the heterogeneity for non-eigen heterogeneous systems can be completely described by $\mathrm{N}$-point correlation functions when $\mathrm{N}$ is infinite. However, a periodic eigen microstructure can be uniquely described by two-point correlation functions (TPCFs). ${ }^{10}$ In general, two-point correlation functions of a d-dimensional isotropic media can be extracted from a $\mathrm{m}$-dimensional cut of the d-dimensional medium $(\mathrm{m}=1$, $2, \ldots, d-1) .{ }^{11}$ However, experimentally, electron microscopy images can be exploited to extract two-point and three-point correlation functions of the microstructures. ${ }^{12-14}$ Smallangle X-ray scattering (SAXS) measurement is another powerful technique capable of providing statistical two-point correlation functions of a large volume of heterogeneous sample without limitation of electron microscopy. ${ }^{15}$

Statistical continuum mechanics methods have been developed as a powerful basis for the reconstruction and 
homogenization of the microstructures. ${ }^{4}$ Weak and strong contrast expansions are two well-known examples of these methods. ${ }^{4}$ Weak contrast expansion is originally developed based on the perturbation series which can perfectly estimate the effective properties for the small contrast of the constituent properties (e.g., less than two). Developing a powerful approach for estimating the effective properties of microstructure with high-contrast constituents led to the strongcontrast expansions. ${ }^{4,16,17}$

In the literature, closed form relations are available to estimate the second and third order bounds on the effective properties of simple microstructures, e.g., coated-sphere Hashin-Shtrikman structures. ${ }^{16}$ However, for more complex microstructure, strong-contrast approximations are usually good alternatives. The d-dimensional strong-contrast formulation was developed to evaluate the effective elastic, electric, thermal, and permeability properties of a microstructure comprising two isotropic phases. ${ }^{4}$ Later, this approach was extended to the multiphase microstructures. ${ }^{16,17}$ Generally, strong-contrast expansions should be evaluated numerically for highly complex structures. For this purpose, the series expansion is usually truncated up to its third order term which only incorporates two-point and three-point correlation functions into the solution. ${ }^{15,18}$ Such truncated expansion is undesirably sensitive to the selection of the reference phase properties.

In the following study, an established strong contrast expansion by $\mathrm{Pham}^{19}$ is adopted and modified to find a third-order estimate of the effective thermal conductivity of multiphase random heterogenous materials. To generalize the applicability of the proposed solution, a recent method $^{20}$ is applied to approximate three point correlation functions from two-point correlation functions of the same microstructure. An approach is also exploited to minimize the effect of reference phase properties on the estimated effective thermal conductivity. As mentioned earlier, the proposed method is suitable for applications in nanomaterials such as CNTs with tubular structure and GNPs with disk-shaped structure. To investigate the validity of the proposed approach, different sets of two-phase and three-phase microstructures comprising tubular and disk-shaped inclusions are selected. The effective thermal conductivity for each microstructure is estimated both via the proposed method and with a standard finite element (FE) method. Finally, the estimated properties of both methods are quantitatively compared and the agreement between the results is discussed.

\section{STRONG-CONTRAST EXPANSION}

For a general microstructure with characteristic microscopic length scale much smaller than that of specimen length scale and consisting of three different phases, where each phase has the volume fraction $\phi_{\alpha}(\alpha=1,2,3)$ and is characterized by isotropic thermal conductivity of $\sigma_{\alpha}$, the local conductivity at position $(\boldsymbol{x})$ is

$$
\sigma(\boldsymbol{x})=\sum_{\alpha=1}^{3} \sigma_{\alpha} \mathcal{I}^{(\alpha)}(\boldsymbol{x})
$$

where the indicator function $\left(\mathcal{I}^{(\alpha)}(\boldsymbol{x})\right.$ is) for each phase is defined by

$$
\mathcal{I}^{(\alpha)}(\boldsymbol{x})= \begin{cases}1 & \boldsymbol{x} \text { in phase } \alpha \\ 0 & \text { otherwise }\end{cases}
$$

Knowing that the local intensity filed $(\boldsymbol{E}(\boldsymbol{x}))$ and local thermal current $(\boldsymbol{J}(\boldsymbol{x}))$ are interrelated by

$$
\boldsymbol{J}(\boldsymbol{x})=\sigma(\boldsymbol{x}) \boldsymbol{E}(\boldsymbol{x}) .
$$

It is possible to embed this microstructure in to a reference medium, with thermal conductivity $\sigma_{0}$, subjected to an intensity field $\boldsymbol{E}_{\mathbf{0}}(\boldsymbol{x})$ at the infinity and define a polarization field $\boldsymbol{P}(\boldsymbol{x})$ by

$$
\boldsymbol{P}(\boldsymbol{x})=\left(\sigma(\boldsymbol{x})-\sigma_{0}\right) \boldsymbol{E}(\boldsymbol{x}) .
$$

Under steady state condition and without sources, the polarization field is related to the temperature distribution (and knowing that $\boldsymbol{E}(\boldsymbol{x})=-\nabla T(\boldsymbol{x}))$ by

$$
\sigma_{0} \Delta T(\boldsymbol{x})=\boldsymbol{\nabla} \cdot \boldsymbol{P}(\boldsymbol{x}) .
$$

By adopting the Green's function solution to the Laplace equation at the infinity, this equation can be solved. However, to obtain the intensity field $\boldsymbol{E}(\boldsymbol{x})$, the solution to this equation should be differentiated by excluding an infinitesimal region around each solution point and introducing a cavity intensity field $(\boldsymbol{F}(\boldsymbol{x}))$. Torquato ${ }^{4}$ has shown that

$$
\boldsymbol{E}(\boldsymbol{x})=\boldsymbol{E}_{\mathbf{0}}(\boldsymbol{x})+\int d x^{\prime} \boldsymbol{G}^{(\mathbf{0})}(\boldsymbol{r}) \cdot \boldsymbol{P}\left(\boldsymbol{x}^{\prime}\right),
$$

where $\boldsymbol{r}=\boldsymbol{x}-\boldsymbol{x}^{\prime}$ and

$$
\boldsymbol{G}^{(\mathbf{0})}(\boldsymbol{r})=-\boldsymbol{D}^{(\mathbf{0})} \delta(\boldsymbol{r})+\boldsymbol{H}^{(\mathbf{0})}(\boldsymbol{r})
$$

and

$$
\boldsymbol{D}^{(\mathbf{0})}=\frac{1}{3 \sigma_{0}} \boldsymbol{I}, \quad \boldsymbol{H}^{(\mathbf{0})}(\boldsymbol{r})=\frac{1}{4 \pi \sigma_{0}} \frac{3 \boldsymbol{n} \boldsymbol{n}-\boldsymbol{I}}{r^{3}},
$$

where the vector $\boldsymbol{n}=\boldsymbol{r} /|\boldsymbol{r}|$, and $\boldsymbol{I}$ is the second order identity tensor. This integral over the surface of an excluded infinitesimal spherical cavity is identically zero, thus, a formulation for the cavity intensity field $F(x)$ can be deduced by substituting Eq. (7) into Eq. (6) as

$$
\boldsymbol{F}(\boldsymbol{x})=\boldsymbol{E}_{\mathbf{0}}(\boldsymbol{x})+\int_{\epsilon} d x^{\prime} \boldsymbol{H}^{(\mathbf{0})}\left(\boldsymbol{x}-\boldsymbol{x}^{\prime}\right) \cdot \boldsymbol{P}\left(\boldsymbol{x}^{\prime}\right)
$$

in which the cavity intensity field is

$$
\boldsymbol{F}(\boldsymbol{x})=\left\{\boldsymbol{I}+\boldsymbol{D}^{(\mathbf{0})}\left[\sigma(\boldsymbol{x})-\sigma_{0}\right]\right\} \cdot \boldsymbol{E}(\boldsymbol{x}) .
$$

By combining Eqs. (10) and (4), the relationship between the polarization field and cavity intensity field is defined by

$$
\boldsymbol{P}(\boldsymbol{x})=L(\boldsymbol{x}) \boldsymbol{F}(\boldsymbol{x}),
$$


where

$$
L(\boldsymbol{x})=3 \sigma_{0} \sum_{\alpha=1}^{3} b_{\alpha 0} \mathcal{I}^{(\alpha)}(\boldsymbol{x})
$$

and

$$
b_{\alpha 0}=\frac{\sigma_{\alpha}-\sigma_{0}}{\sigma_{\alpha}+2 \sigma_{0}}, \quad \alpha=1,2,3 .
$$

Knowing that the average cavity field is related to the polarization field by

$$
\langle\boldsymbol{P}(\boldsymbol{x})\rangle=\boldsymbol{L}_{\boldsymbol{e}} \cdot\langle\boldsymbol{F}(\boldsymbol{x})\rangle,
$$

in which the angular bracket represents an ensemble average. With further mathematical manipulations, it is possible to show that the second-order tensor $\boldsymbol{L}_{e}$ is related to the effective thermal conductivity tensor for the microstructure $\left(\boldsymbol{\sigma}_{\boldsymbol{e}}\right)$ by

$$
\boldsymbol{L}_{\boldsymbol{e}}=3 \sigma_{0}\left\{\boldsymbol{\sigma}_{\boldsymbol{e}}-\sigma_{0} \boldsymbol{I}\right\} \cdot\left\{\boldsymbol{\sigma}_{\boldsymbol{e}}+2 \sigma_{0} \boldsymbol{I}\right\}^{-1} .
$$

Torquato ${ }^{4}$ has shown the explicit form of the effective thermal conductivity tensor to be

$$
\begin{aligned}
\boldsymbol{L}_{\boldsymbol{e}}^{-1}(\mathbf{1})= & \frac{\boldsymbol{I}(1)}{\langle L(1)\rangle}-\int\left(\frac{\langle L(1) L(2)\rangle-\langle L(1)\rangle\langle L(2)\rangle}{\langle L(1)\rangle\langle L(2)\rangle}\right) \boldsymbol{H}^{(0)}(1,2) d 2 \\
& -\iint\left(\frac{\langle L(1) L(2) L(3)\rangle}{\langle L(1)\rangle\langle L(2)\rangle}-\frac{\langle L(1) L(2)\rangle\langle L(2) L(3)\rangle}{\langle L(1)\rangle\langle L(2)\rangle\langle L(3)\rangle}\right) \boldsymbol{H}^{(0)}(1,2) \cdot \boldsymbol{H}^{(0)}(2,3) d 2 d 3+\cdots,
\end{aligned}
$$

in this equation, the shorthand notation $x=1, x^{\prime}=2$ and $\boldsymbol{x}^{\prime \prime}=3$ were utilized. This equation utilizes $\langle L(1), \ldots, L(n)\rangle$ terms which are related to the $\mathrm{N}$-point microstructural correlation functions. For instance, for a combination of three phases $\alpha, \beta$, and $\gamma$ one can show that

$$
\begin{gathered}
\langle L(1)\rangle=3 \sigma_{0} \sum_{\alpha=1}^{3} b_{\alpha 0} S_{1}^{(\alpha)}(1), \\
\langle L(1) L(2)\rangle-\langle L(1)\rangle\langle L(2)\rangle=\left(3 \sigma_{0}\right)^{2} \sum_{\alpha=0}^{3} \sum_{\beta=0}^{3} b_{\alpha 0} b_{\beta 0}\left[S_{2}^{(\alpha \beta)}(1,2)\right. \\
\left.-S_{1}^{(\alpha)}(1) S_{1}^{(\beta)}(2)\right]
\end{gathered}
$$

in which $S_{3}^{(\alpha \beta \gamma)}$ is a 3-point correlation function defined by

$S_{3}^{(\alpha \beta \gamma)}\left(\boldsymbol{x}_{\mathbf{1}}, \boldsymbol{x}_{\mathbf{2}}, \boldsymbol{x}_{\mathbf{3}}\right)=\left\langle\mathcal{I}^{(\alpha)}\left(\boldsymbol{x}_{\mathbf{1}}\right) \mathcal{I}^{(\beta)}\left(\boldsymbol{x}_{\mathbf{2}}\right) \mathcal{I}^{(\gamma)}\left(\boldsymbol{x}_{\mathbf{3}}\right)\right\rangle, \quad \alpha, \beta, \gamma=1,2,3$.

\section{GENERAL ANISOTROPIC SOLUTION}

Following Torquato, ${ }^{4}$ Pham,${ }^{16}$ and Quang, ${ }^{19}$ it is possible to approximate the series in Eq. (16) up to its third-order terms and then by substituting Eq. (15) into it, after some simplifications, the effective conductivity tensor for a general case of macroscopically anisotropic microstructure with three phases reduces to

$$
\left(\frac{1}{\sigma_{0}} \boldsymbol{\sigma}_{\boldsymbol{e}}-\boldsymbol{I}\right)^{-1}=\frac{1}{3}\left(\frac{1}{\sum_{\alpha=1}^{3} \phi_{\alpha} b_{\alpha 0}}-1\right) \boldsymbol{I}-\boldsymbol{A}_{\mathbf{2}}-\boldsymbol{A}_{\mathbf{3}} .
$$

In this equation, $\boldsymbol{A}_{\mathbf{2}}$ and $\boldsymbol{A}_{\mathbf{3}}$ are second-order and third-order terms, respectively. Using Eqs. (17) and (18) and similar expansion for higher order terms, one can show that

$$
\begin{aligned}
\boldsymbol{A}_{\mathbf{2}}= & \frac{\sigma_{0}}{\left(\sum_{\alpha=1}^{3} \phi_{\alpha} b_{\alpha 0}\right)^{2}} \int \sum_{\alpha=1}^{3} \sum_{\beta=1}^{3}\left(b _ { 0 \alpha } b _ { 0 \beta } \left[S_{2}^{(\alpha \beta)}(1,2)\right.\right. \\
& \left.\left.-S_{1}^{(\alpha)}(1) S_{1}^{(\beta)}(2)\right]\right) \boldsymbol{H}^{(\mathbf{0})}(\mathbf{1}, \mathbf{2}) d 2,
\end{aligned}
$$

It should be noted that in both Eqs. (21) and (22), $b_{0 \alpha}(\alpha=1,2,3)$ terms only depend on the properties of each phase and the reference medium and, therefore, the summa- tions can be expanded and these terms can be factored out of each tensor integration as constants. The third-order terms require three-point correlation function which can be 


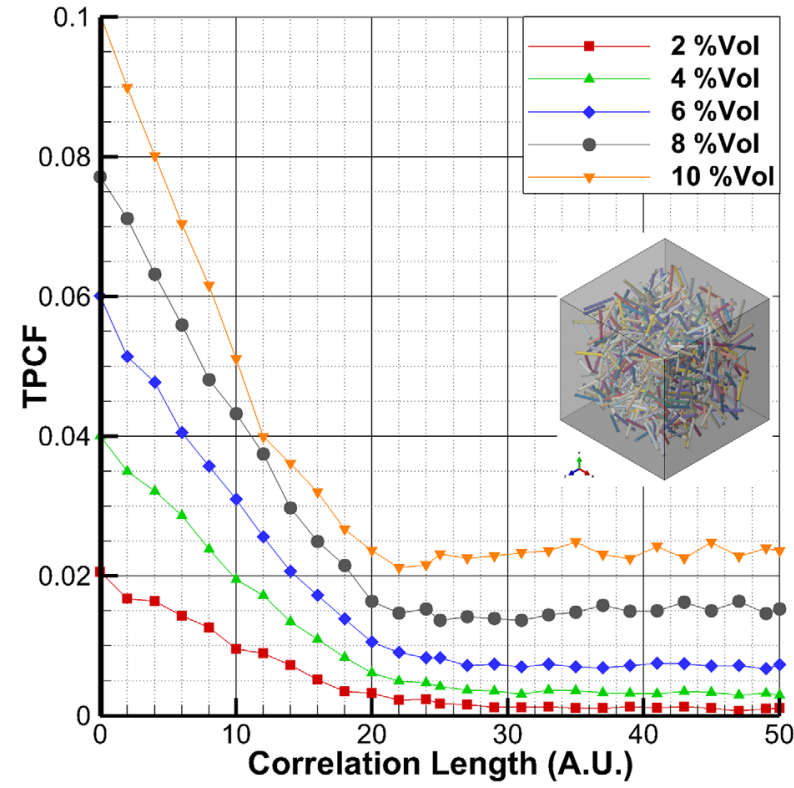

FIG. 1. TPCF of isotropic homogeneous microstructures loaded with 2-10 vol. $\%$ tubular inclusions $(\phi=20, l=200$, and aspect ratio $=10)$. The inset shows $3 \mathrm{D}$ view of the RVE for 4 vol. \% configuration.

approximated properly; the details are addressed in the next section. Finally, it should be noted that for a general microstructure, Eq. (20) has no closed form analytical solution and therefore it should be evaluated numerically. Because of the truncated nature of this expansion, care must be taken during the numerical solution in order to guarantee its convergence to the true solution. Detailed discussion about the numerical evaluation of this equation is provided later in this article.

\section{MICROSTRUCTURAL DESCRIPTOR FUNCTIONS}

Several microstructural descriptors are developed in numerous fields of applied physics. Among them, the $\mathrm{N}$-point correlation functions (or probability functions) introduced by Eq. (19) have been widely applied in the context of determining the effective properties. ${ }^{4}$ TPCFs are the lowest order of their kind that are capable of providing geometrical information about a unique microstructure. ${ }^{4}$ For instance, Figure 1 shows TPCF of the homogeneous two-phase microstructures with isotropic dispersion of unique tubular inclusions calculated numerically from a representative volume element (RVE). The RVEs were generated by the method described elsewhere. ${ }^{21}$ As the correlation length $\left(\left|\boldsymbol{x}-\boldsymbol{x}^{\prime}\right|\right)$ approaches infinity, TPCF is converging to a constant value $\left(\approx \phi_{\alpha}^{2}\right)$ with no dependence on the orientation.

Similar functions for an anisotropic microstructure with tubular inclusions aligned in $\mathrm{x}$-direction should show strong correlation in this direction compared to the other directions. Therefore, in order to correctly represent this microstructure, a three dimensional correlation function is required. Corson method $^{22}$ is applied here to evaluate the three dimensional TPCF of this microstructure as illustrated in Figure 2.

Analytical evaluation of TPCFs is only limited to certain simple microstructures. ${ }^{4}$ Direct numerical evaluation of higher order correlation functions is an expensive computational process. Different methods are developed to approxi-

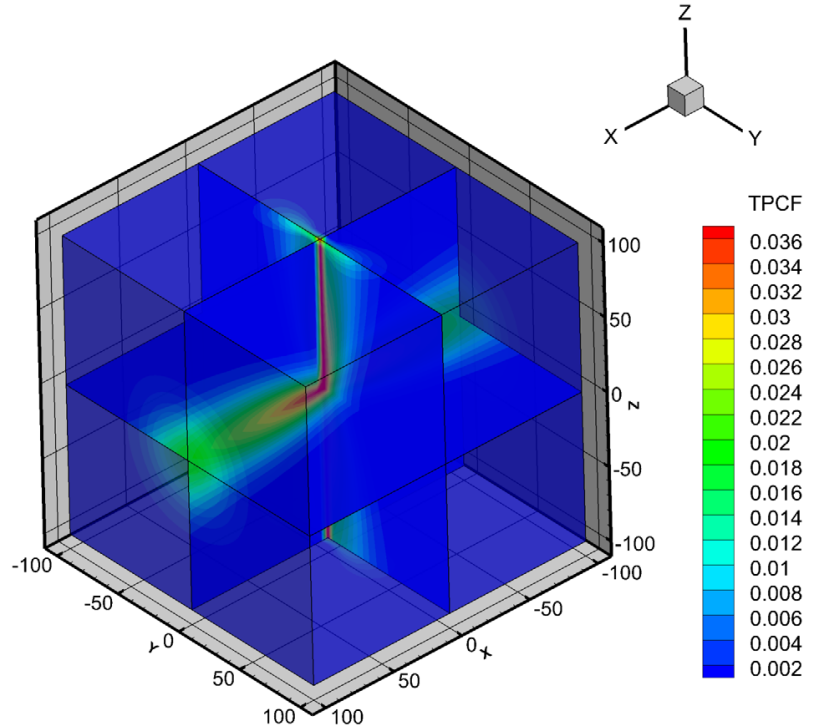

FIG. 2. Three dimensional representation of a two-point correlation function of homogeneous anisotropic microstructure loaded with 4 vol. \% tubular inclusions $(\phi=20, l=200$, and aspect ratio $=10)$ with preference orientation in the $\mathrm{x}$-direction.

mate higher order probability functions from lower order ones. $^{20,23}$ Recently, Baniassadi et al. ${ }^{20}$ introduced a method to approximate $\mathrm{N}$-point correlation functions from $(\mathrm{N}-1)$ point correlation functions. Similar approach is employed here to approximate three-point correlation functions by two-point functions according to

$$
\begin{aligned}
S_{3}^{(\alpha \beta \gamma)}(1,2,3) \approx & W_{1}^{3} \frac{S_{2}^{(\alpha \beta)}(1,2) S_{2}^{(\alpha \gamma)}(1,3)}{S_{1}^{(\alpha)}(1)} \\
& +W_{2}^{3} \frac{S_{2}^{(\alpha \beta)}(1,2) S_{2}^{(\beta \gamma)}(2,3)}{S_{1}^{(\beta)}(2)} \\
& +W_{3}^{3} \frac{S_{2}^{(\beta \gamma)}(2,3) S_{2}^{(\alpha \gamma)}(1,3)}{S_{1}^{(\gamma)}(3)}
\end{aligned}
$$

in which

$$
W_{i}^{3}=\frac{R_{i}}{\sum_{j=1}^{3} R_{j}}, \quad i=1,2,3
$$

where $R_{1}, R_{2}$, and $R_{3}$ are the shortest distances between each pair of points (1-3).

\section{REFERENCE MEDIUM SELECTION}

The strong contrast expansion introduced in Eq. (16) is based on the assumption that the specimen is completely bounded by a reference medium at infinity. For the limit of infinite number of terms, the two phase expansion is known to converge to the true solution regardless of the properties of the reference medium $\left(\sigma_{0}\right){ }^{4}$ However, for a three-terms truncation, the absolute convergence to the true solution is contestable. In fact, when the properties of the reference medium $\left(\sigma_{0}\right)$ are close enough to the effective properties, there is a better chance for this equation to converge to the true 


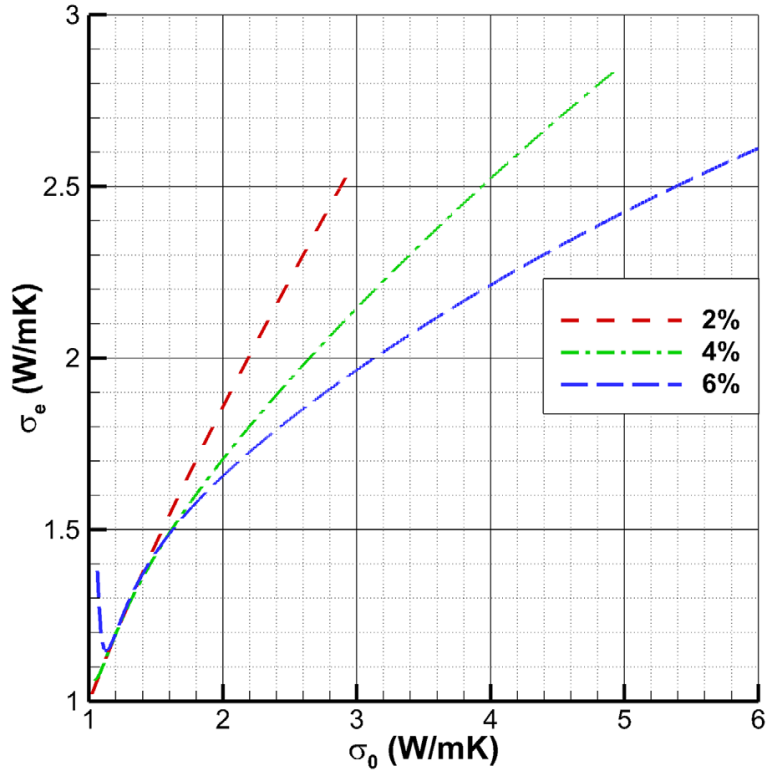

FIG. 3. Effect of the reference phase $\left(\sigma_{0}\right)$ selection on the effective conductivity for the microstructures loaded with 2, 4 and 6 vol. \% tubular inclusions $\left(\right.$ aspect ratio $=10, \sigma_{\text {tube }}=100$, and $\left.\sigma_{\text {matrix }}=1\right)$.

solution. For only two phase media, Torquato ${ }^{4}$ recommended one of the constituents to be selected as the reference medium for the solution. Even under such conditions, two different expansions can be written with no guarantee for a single solution. For a microstructure comprising more than two phases, with orders of magnitude discrepancy between their properties, the effective properties can be significantly different from each of the individual phases. For instance, the effective conductivity of a microstructure composed of isotropically dispersed tubular inclusions inside a matrix phase with two orders of magnitude difference between their thermal conductivities $\left(\sigma_{1} / \sigma_{2}=100\right)$ is shown in Figure 3. It can be observed that by varying the thermal conductivity of the reference medium between the arithmetic and harmonic averages, there is a three-fold variation in the estimation of the effective thermal conductivity of the microstructure. This observation strengthens the necessity to devise a method for proper selection of the reference phase properties.

In order to find proper interval for $\sigma_{0}$, one can solve Eq. (20) for $\sigma_{e}$

$$
\begin{aligned}
\boldsymbol{\sigma}_{\boldsymbol{e}} & =\sigma_{0}\left(\boldsymbol{I}+\left(\frac{1}{3}\left(\frac{1}{\sum_{\alpha=1}^{3} \phi_{\alpha} b_{\alpha 0}}-1\right) \boldsymbol{I}-\boldsymbol{A}_{\mathbf{2}}-\boldsymbol{A}_{\mathbf{3}}\right)^{-1}\right) \\
& =\boldsymbol{f}\left(\sigma_{0}, \text { microstructure }\right),
\end{aligned}
$$

in which $f$ represents the approximation function. Assuming the true solution is $\sigma_{\text {true }}$, the absolute approximation error as a function of $\sigma_{0}$ is defined by

$$
\boldsymbol{e}\left(\sigma_{0}\right)=\left(\boldsymbol{f}\left(\sigma_{0}\right)-\boldsymbol{\sigma}_{\text {true }}\right)^{2} .
$$

Assuming the function $\boldsymbol{e}$ is continuous and differentiable through the domain, one can differentiate this equation and set it to zero as

$$
\frac{\partial \boldsymbol{e}}{\partial \sigma_{0}}=2 \frac{\partial \boldsymbol{f}}{\partial \sigma_{0}}\left(\boldsymbol{f}\left(\sigma_{0}\right)-\boldsymbol{\sigma}_{\text {true }}\right)=0 .
$$

The solutions to this equation is

$$
\begin{gathered}
\frac{\partial \boldsymbol{f}}{\partial \sigma_{0}}=0, \\
\boldsymbol{f}\left(\sigma_{0}\right)=\boldsymbol{\sigma}_{\text {true }} .
\end{gathered}
$$

The roots of these equations poses minimal sensitivity of the function $\boldsymbol{e}$ to the reference phase properties. Equation (29) is trivial, however, Eq. (28) can be solved with available information. Thus, the roots of Eq. (28) are the best candidates for $\sigma_{0}$. Considering second order continuity for Eq. (26), it can be differentiated one more time such that

$$
\frac{\partial^{2} \boldsymbol{e}\left(\sigma_{0}\right)}{\partial \sigma_{0}^{2}}=2 \frac{\partial^{2} \boldsymbol{f}}{\partial \sigma_{0}^{2}}\left(\boldsymbol{f}\left(\sigma_{0}\right)-\boldsymbol{\sigma}_{\text {true }}\right)+2\left(\frac{\partial \boldsymbol{f}}{\partial \sigma_{0}}\right)^{2},
$$

the second term of this equation vanishes at the candidate points. Intuitively, the solutions of Eq. (25) at the candidate points either overestimate or underestimate the effective properties. Based on this fact, the expression inside the parenthesis of the first term in this equation is either positive or negative and, therefore, the best approximation to the effective properties is either local minima or maxima of Eq. (25). When there is at least one local minimum and one maximum point available, the arithmetic average value of them could offer a better estimate of the effective properties by canceling out errors.

Evaluation of Eq. (27) analytically is only viable for very simple microstructures. However, numerically, it can be evaluated potentially for any combination of microstructure and properties. For the isotropic microstructures described earlier (Figure 1), Figure 4 is showing variation of $\sigma_{e}$ $\left(\sigma_{\boldsymbol{e}}=\sigma_{e} \boldsymbol{I}\right)$ with $\sigma_{0}$. The local minimum and maximum are

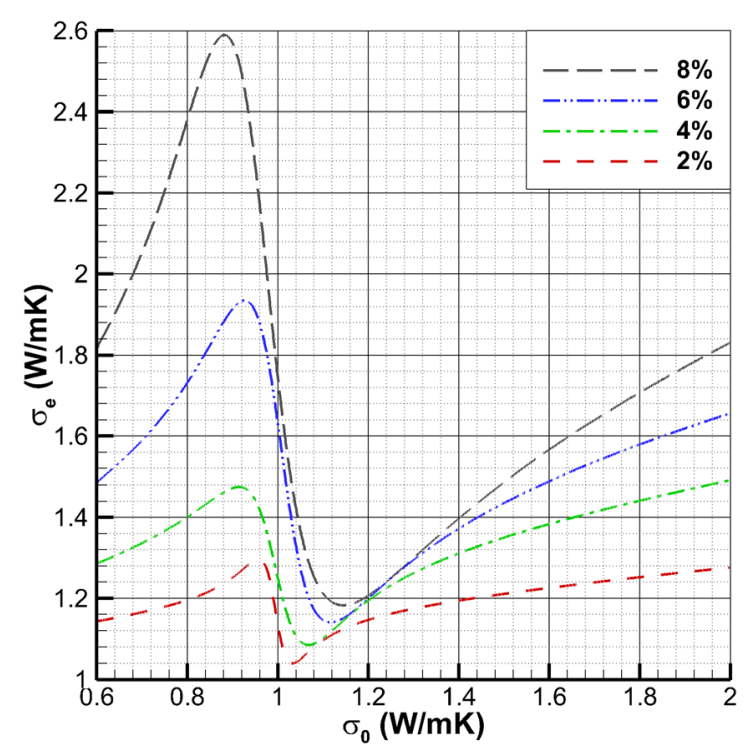

FIG. 4. Candidate points for the evaluation of $\sigma_{e}$ in an isotropic microstructure loaded with $2,4,6$, and 8 vol. $\%$ tubular inclusions (aspect ratio $=10$, $\sigma_{\text {tube }}=100$, and $\sigma_{\text {matrix }}=1$ ). 


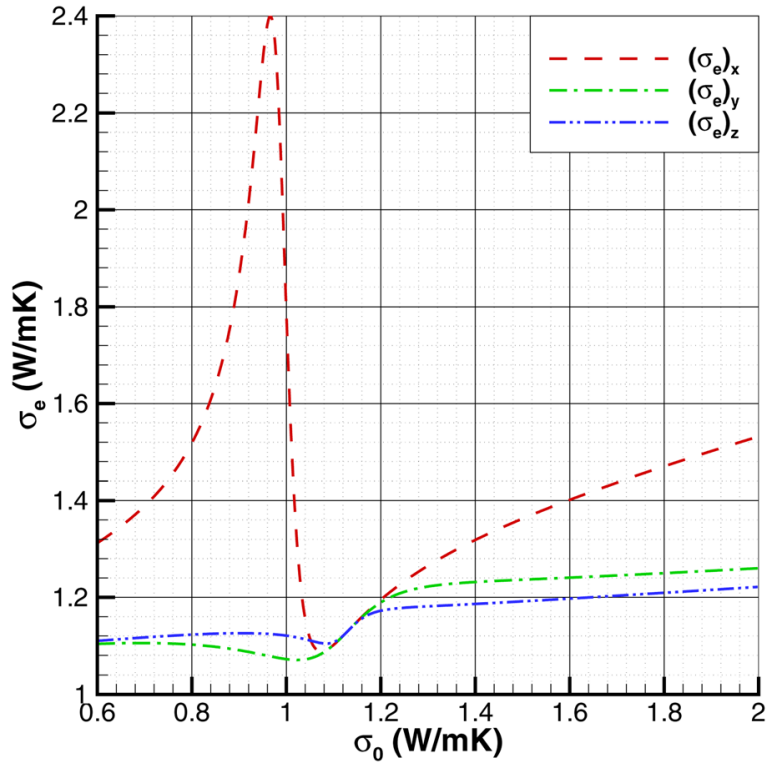

FIG. 5. Existence of different candidate points for an anisotropic microstructure loaded with 4 vol. \% tubular inclusions with preferred orientation in the $\mathrm{x}$-direction (aspect ratio $=10, \sigma_{\text {tube }}=100$, and $\sigma_{\text {matrix }}=1$ ).

providing an upper estimate and a lower estimate of the effective properties.

For a general anisotropic microstructure, Eq. (27) yields different candidate points. Each of these points represents a possible estimate of the effective properties. For instance, Figure 5 shows these points for the anisotropic microstructure described earlier in Figure 2.

\section{VALIDATION STUDY}

In order to establish the validity of the proposed method, the effective thermal conductivity for three phase microstructures comprising tubular inclusion, disk-shaped inclusions, and a combination of both are studied. A RVE of the microstructures was generated by stochastic Monte Carlo method through random sequential addition process as described elsewhere. ${ }^{24}$ Strong contrast predictions were computed from two-point correlation function of the microstructure and approximated three-point correlation functions. For all microstructures, the effective thermal conductivity was compared to that calculated through finite element simulations using commercial finite element code Abaqus

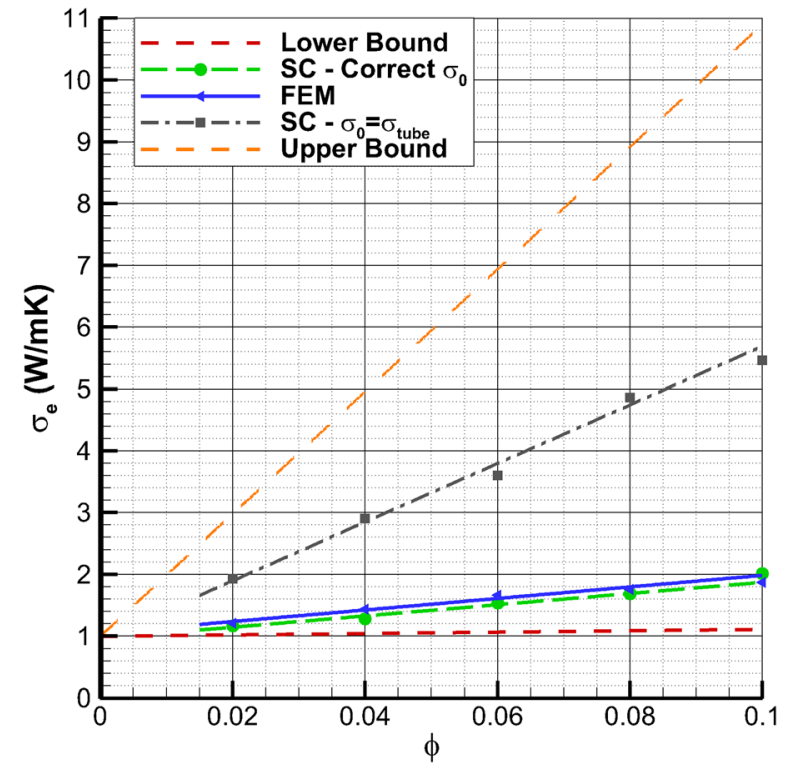

FIG. 7. Comparison between the effective thermal conductivities predicted by the strong contrast method and the finite element method for isotropic microstructures loaded with tubular inclusions (aspect ratio $=10$ and $\sigma_{\text {tube }}=100$ and $\left.\sigma_{\text {matrix }}=1\right)$.

(version 6.10-EF). For the finite element model, the microstructure was simulated and meshed with four node tetrahedral elements (DC3D4) under the presumption of perfect bounding between the particulate inclusions and the matrix. In order to ensure the convergence of the FE solution, a number of mesh refinement strategies were carried out as well as preliminary local mesh refinement around each inclusion and global mesh refinement in the convergence study. The boundary conditions were chosen to be constant temperatures uniformly distributed over the mesh grids in two opposing side ( 273 and $373 \mathrm{~K}$ ) of the simulation cuboid and the steady state solution was evaluated. Finally, the effective thermal conductivity was calculated from the temperature difference and the volume-averaged heat flux vector over the whole cuboid. Figure 6 shows the output of one of the finite elements simulations for a microstructure with disk-shaped inclusions. Depending on the volume fraction of the inclusions, and considering the solution convergence study, each FE simulation was taking between 10 and $96 \mathrm{~h}$ on a hexacore windows based machine with 12 GB of memory.

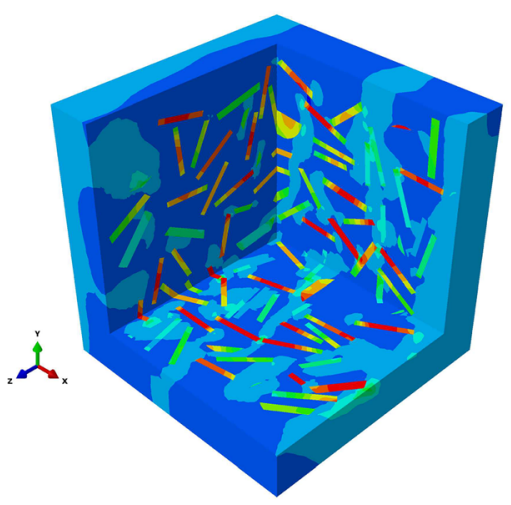

(a)

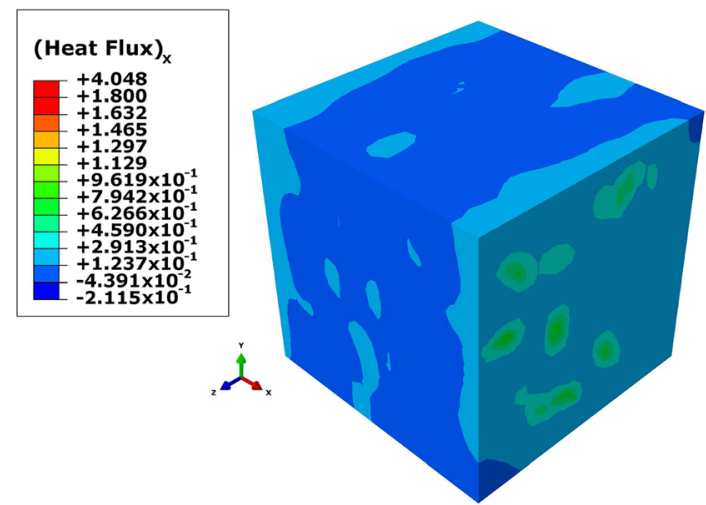

(b)
FIG. 6. FE calculation of the distribution of the heat-flux in $x$-direction in the RVE loaded with 10 vol. \% disk-shaped inclusions (aspect ratio $=10, \sigma_{\text {disk }}=100$, and $\sigma_{\text {matrix }}=1$ ) (a) internal view (b) full view. 


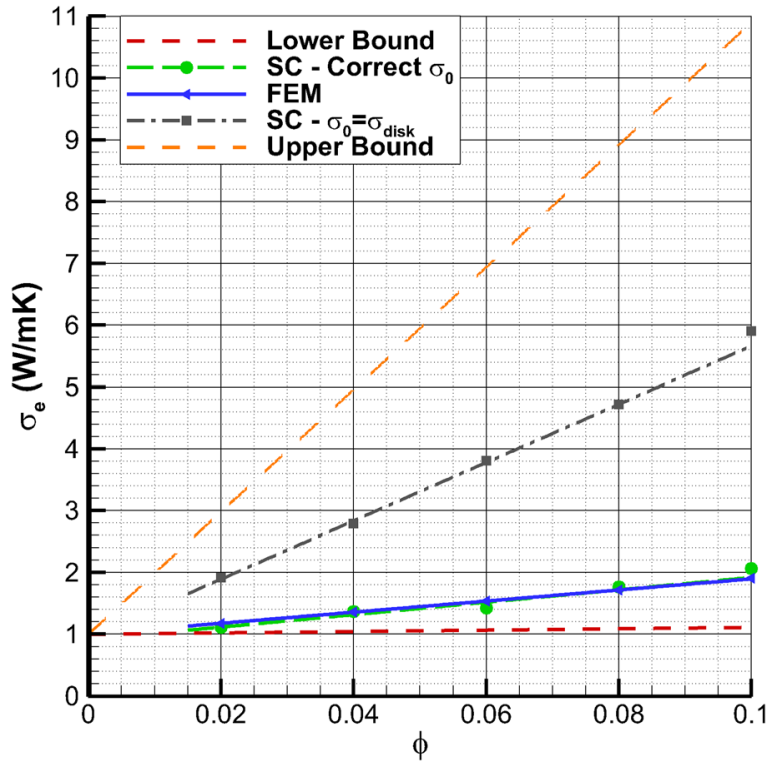

FIG. 8. Comparison between the effective thermal conductivities predicted by the strong contrast method and the finite element method for isotropic microstructures loaded with disk-shaped inclusions (aspect ratio $=10$, $\sigma_{\text {disk }}=100$, and $\sigma_{\text {matrix }}=1$ ).

Figure 7 is showing the effective thermal conductivity for the homogeneous composite specimens loaded with different volume fractions of the tubular inclusions and with the microstructure described in Figure 1. The thermal conductivity of the isotropic inclusions was selected to be hundred times higher than that for matrix phase $\left(\sigma_{\text {tube }} / \sigma_{\text {matrix }}=100\right)$. In this figure, the strong contrast predictions are calculated twice. Initially, the reference medium was selected to be identical to the inclusion phase and then it was selected by the proposed method. Both predictions are also compared with the lower-bound and the FE predictions. It can be

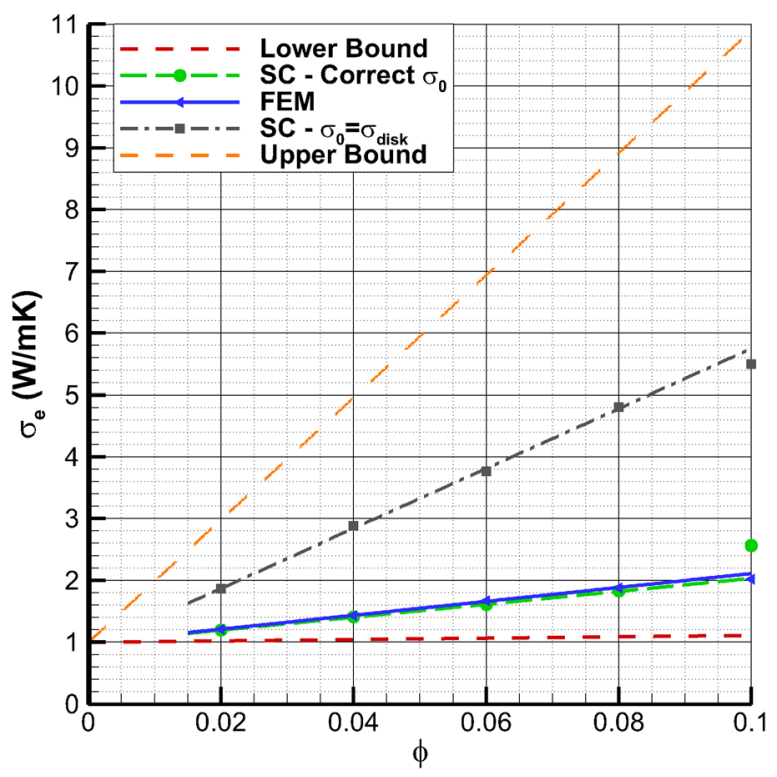

FIG. 9. Comparison between the effective thermal conductivities predicted by the strong contrast method and the finite element method for the microstructures loaded with tubular and disk-shaped inclusions (aspect ratio $=10$, $\phi_{\text {tube }}=\phi_{\text {disk }}, \sigma_{\text {disk }}=\sigma_{\text {tube }}=100$, and $\sigma_{\text {matrix }}=1$ ).

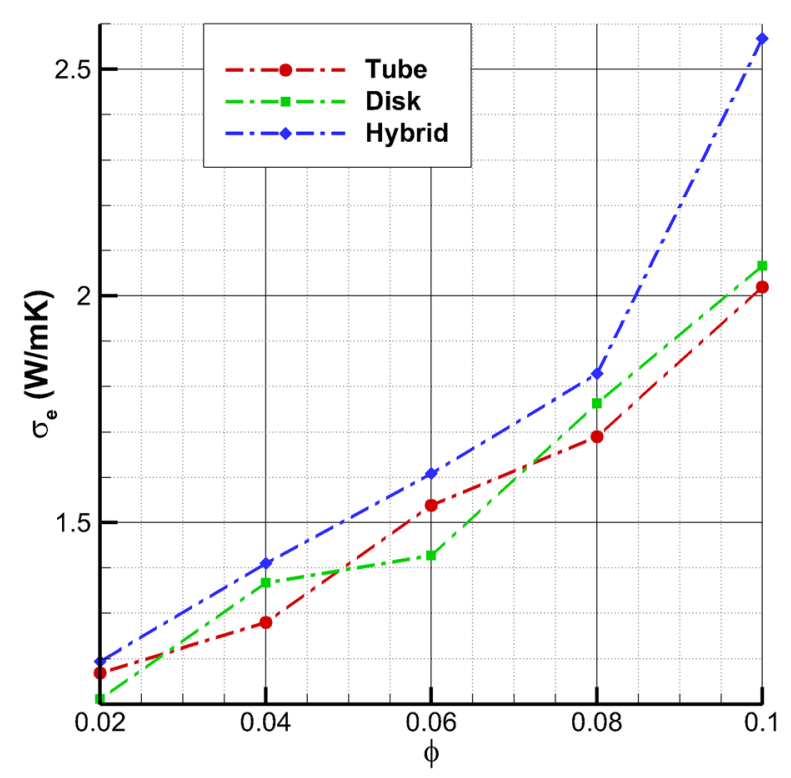

FIG. 10. Proposed strong contrast method predicts the synergetic improvement in the effective thermal conductivity by tube-disk hybridization into isotropic microstructure. (aspect ratio $=10, \quad \phi_{\text {tube }}=\phi_{\text {disk }}$, $\sigma_{\text {disk }}=\sigma_{\text {tube }}=100$, and $\sigma_{\text {matrix }}=1$ ).

observed that only the predictions of the proposed method are in good agreement with finite elements results in the case of tubular inclusions.

Similarly, the effective conductivity of the homogeneous microstructures filled with disk-shaped inclusions was also evaluated. Figure 8 shows the comparison between the predicted results of the different methods for various volume fractions of disk-shaped inclusions. Good agreement with finite element predictions is also observed for the proposed method.

A number of multiphase hybrid disk-tube microstructures were also studied. In all cases, microstructures with homogenous distribution of the disk and the tube phases with equal volume fractions were analyzed. Figure 9 compares the effective thermal conductivity of these hybrid microstructures. Again, perfect agreement is observed between the finite element predictions and the proposed method predictions.

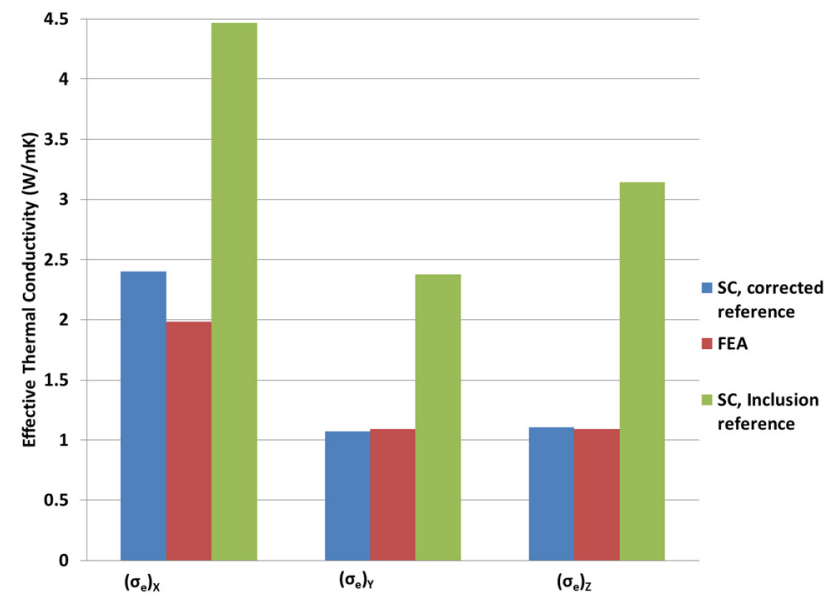

FIG. 11. Effective thermal conductivity of the anisotropic microstructure loaded with tubular inclusions with preferential orientation in the x-direction $\left(\right.$ aspect ratio $=10, \sigma_{\text {tube }}=100$, and $\left.\sigma_{\text {matrix }}=1\right)$. 
Figure 10 compares the strong contrast predictions for all three configurations. It can be observed that disk-tube hybridization improves the effective conductivity over that for each individual phase. This synergetic enhancement in the properties was also observed in the corresponding finite element study.

Finally, the method was applied for the anisotropic microstructure loaded with tubular inclusions as described in Figure 2. Figure 11 compares the effective thermal conductivity tensor. It can be observed that the proposed method is in good agreement with the finite element results.

\section{CONCLUSIONS}

In this study, a modified version of the strong contrast expansions is formulated to evaluate the effective thermal conductivity of multiphase heterogenous materials. The effect of the reference medium on the effective thermal conductivity is discussed both analytically and numerically. A method is proposed to minimize the effect of the reference medium on the solution. The proposed method formulates error between the predicted thermal conductivity and the true thermal conductivity as a function of the reference phase properties and then finds its extremal points. It is argued that by choosing the properties of the reference phase on the neighborhood of these extremal points, the predicted effective properties will contain minimal error compared to the practice of arbitrary selection of the reference phase followed by existing methods. In order to establish the validity of the proposed method, its prediction is compared with those from finite element simulations for three different microstructures consisting of tubular, disk-shaped inclusions, and a combination of both. Good agreements between the proposed method predictions and the FE calculations are observed for all three different microstructure cases. Furthermore, this study suggests that-compared to the single inclusion scenario-a synergistic combination of tube and disk-shaped inclusions can further enhance the effective thermal conductivity of a hybrid microstructure.

\section{ACKNOWLEDGMENTS}

This work has been supported by the Office of Naval Research (ONR) Grant No. 10960991 and the National Science Foundation (NSF) award CMMI: Career-0846589. The authors gratefully acknowledge this support.

${ }^{1}$ K. I. Winey and R. A. Vaia, MRS Bull. 32, 314 (2007).

${ }^{2}$ B. Jang and A. Zhamu, J. Mater. Sci. 43, 5092 (2008).

${ }^{3}$ D. Bigg, in Thermal and Electrical Conductivity of Polymer Materials, Advances in Polymer Science Vol. 119 (Springer, Berlin, 1995), p. 1.

${ }^{4}$ S. Torquato, Random Heterogeneous Materials (Springer, 2005).

${ }^{5}$ T. Chen, G. J. Dvorak, and Y. Benveniste, ASME Trans. J. Appl. Mech. 59, 539 (1992).

${ }^{6}$ R. Guinovart-Daz, R. Rodrguez-Ramos, J. Bravo-Castillero, F. J. Sabina, J. A. Otero-Hernndez, and G. A. Maugin, Mech. Mater. 37, 1119 (2005).

${ }^{7}$ M. Hori and S. Munasighe, Mech. Mater. 31, 471 (1999).

${ }^{8}$ J. C. H. Affdl and J. L. Kardos, Polym. Eng. Sci. 16, 344 (1976).

${ }^{9}$ M. J. Beran, Statistical Continuum Theories, Monographs in Statistical Physics and Thermodynamics (Interscience, New York, 1968).

${ }^{10}$ M. G. Rozman and M. Utz, Phys. Rev. Lett. 89, 135501 (2002).

${ }^{11}$ S. Torquato, J. Chem. Phys. 111, 8832 (1999).

${ }^{12}$ M. Baniassadi, H. Garmestani, D. Li, S. Ahzi, M. Khaleel, and X. Sun, Acta Mater. 59, 30 (2011).

${ }^{13}$ A. Ghazavizadeh, N. Soltani, M. Baniassadi, F. Addiego, S. Ahzi, and H. Garmestani, Mech. Mater. 51, 88 (2012).

${ }^{14}$ H. A. Hamedani, M. Baniassadi, M. Khaleel, X. Sun, S. Ahzi, D.Ruch, and H. Garmestani, J. Power Sources 196, 6325 (2011).

${ }^{15}$ M. Baniassadi, F. Addiego, A. Laachachi, S. Ahzi, H. Garmestani, F. Hassouna, A. Makradi, V. Toniazzo, and D. Ruch, Acta Mater. 59, 2742 (2011).

${ }^{16}$ D. C. Pham and S. Torquato, J. Appl. Phys. 94, 6591 (2003).

${ }^{17}$ S. Torquato, Phys. Rev. Lett. 79, 681 (1997).

${ }^{18}$ M. Baniassadi, A. Laachachi, F. Hassouna, F. Addiego, R. Muller, H. Garmestani, S. Ahzi, V. Toniazzo, and D. Ruch, Compos. Sci. Technol. 71, 1930 (2011)

${ }^{19}$ H. Le Quang, G. Bonnet, and D. C. Pham, Phys. Rev. E 84, 061153 (2011).

${ }^{20}$ M. Baniassadi, S. Ahzi, H. Garmestani, D. Ruch, and Y. Remond, J. Mech. Phys. Solids 60, 104 (2012).

${ }^{21}$ M. Safdari and M. Al-Haik, Nanotechnology 23, 405202 (2012).

${ }^{22}$ P. B. Corson, J. Appl. Phys. 45, 3165 (1974).

${ }^{23}$ A. Mikdam, A. Makradi, S. Ahzi, H. Garmestani, D. Li, and Y. Remond, J. Mech. Phys. Solids 57, 76 (2009).

${ }^{24}$ M. Baniassadi, M. Safdari, A. Ghazavizadeh, H. Garmestani, S. Ahzi, J. Grcio, and D. Ruch, J. Phys. D: Appl. Phys. 44, 455306 (2011). 